\title{
BMJ Open Computerised cognitive training to improve cognition including delirium following coronary artery bypass grafting surgery: protocol for a blinded randomised controlled trial
}

Danielle Greaves (1) , ${ }^{1}$ Peter J Psaltis, ${ }^{2,3,4}$ Amit Lampit, ${ }^{5,6}$ Daniel H J Davis, ${ }^{7}$ Ashleigh E Smith, ${ }^{8}$ Alice Bourke, ${ }^{9}$ Michael G Worthington, ${ }^{10}$ Michael J Valenzuela, ${ }^{11}$ Hannah A D Keage ${ }^{1}$

To cite: Greaves D, Psaltis PJ, Lampit A, et al. Computerised cognitive training to improve cognition including delirium following coronary artery bypass grafting surgery: protocol for a blinded randomised controlled trial. BMJ Open 2020;10:e034551. doi:10.1136/ bmjopen-2019-034551

\section{- Prepublication history and} additional material for this paper are available online. To view these files, please visit the journal online (http://dx.doi. org/10.1136/bmjopen-2019034551).

Received 25 September 2019 Revised 05 December 2019 Accepted 14 January 2020
Check for updates

(c) Author(s) (or their employer(s)) 2020. Re-use permitted under CC BY-NC. No commercial re-use. See rights and permissions. Published by BMJ.

For numbered affiliations see end of article.

Correspondence to Dr Hannah A D Keage; Hannah.Keage@unisa.edu.au

\section{ABSTRACT}

Introduction Coronary artery bypass grafting (CABG) surgery is known to improve vascular function and cardiac-related mortality rates; however, it is associated with high rates of postoperative cognitive decline and delirium. Previous attempts to prevent post-CABG cognitive decline using pharmacological and surgical approaches have been largely unsuccessful. Cognitive prehabilitation and rehabilitation are a viable yet untested option for $\mathrm{CABG}$ patients. We aim to investigate the effects of preoperative cognitive training on delirium incidence, and preoperative and postoperative cognitive training on cognitive decline at 4 months post-CABG.

Methods and analysis This study is a randomised, single-blinded, controlled trial investigating the use of computerised cognitive training (CCT) both pre-CABG and post-CABG (intervention group) compared with usual care (control group) in older adults undergoing CABG in Adelaide, South Australia. Those in the intervention group will complete 1-2 weeks of CCT preoperatively (45-60 min sessions, 3.5 sessions/week) and 12 weeks of CCT postoperatively (commencing 1 month following surgery, 45-60 min sessions, 3 sessions/week). All participants will undergo cognitive testing preoperatively, over their hospital stay including delirium, and postoperatively for up to 1 year. The primary delirium outcome variable will be delirium incidence (presence vs absence); the primary cognitive decline variable will be at 4 months (significant decline vs no significant decline/improvement from baseline). Logistic regression modelling will be used, with age and gender as covariates. Secondary outcomes include cognitive decline from baseline to discharge, and at 6 months and 1 year post-CABG.

Ethics and dissemination Ethics approval was obtained from the Central Adelaide Local Health Network Human Research Ethics Committee (South Australia, Australia) and the University of South Australia Human Ethics Committee, with original approval obtained on 13 December 2017. It is anticipated that approximately two to four publications and multiple conference presentations (national and international) will result from this research.
Strengths and limitations of this study

- This study will be the first to deliver cognitive training preoperatively and postoperatively to older patients undergoing coronary artery bypass grafting (CABG) surgery.

- The results of this study may provide a prevention strategy for cognitive impairments, including delirium, observed post-CABG surgery.

- We are limited by a two-group design (control vs intervention) due to feasibility issues; therefore, the effects of only pre-CABG and only post-CABG cognitive training cannot be investigated.

- The generalisability of the study will be limited to Adelaide (South Australia) metropolitan CABG patients.

Trial registration number This clinical trial is registered with the Australian New Zealand Clinical Trials Registry and relates to the pre-results stage. Registration number: ACTRN12618000799257.

\section{INTRODUCTION}

Coronary artery bypass grafting (CABG) is one of the main treatments used to revascularise patients suffering from severe coronary artery disease, where coronary vessels are dangerously stenosed due to atherosclerotic plaque build-up. ${ }^{1}$ Coronary artery narrowing commonly results in myocardial ischaemia, which affects cardiac output. This can lead to reduced blood perfusion of the brain, contributing to cognitive impairment. ${ }^{2}$ The cognitive domains most affected by the presence of coronary artery disease are psychomotor speed, memory and executive function. ${ }^{3}$ Impairments in these domains are characteristic of vascular dementia, as similar pathological mechanisms are likely at play. ${ }^{4}$ 
While CABG surgery is known to improve cardiac function and mortality, ${ }^{5}$ CABG is also associated with a high risk of developing postoperative cognitive impairment. A recent meta-analysis by our group investigated cognitive outcomes of CABG patients using a crosssectional approach (percentage at specific timepoints) and reported that postoperative cognitive impairment was present in $43 \%$ of patients acutely (up to 4 days postoperatively). ${ }^{6}$ This was lower $(25 \%)$ in patients $6-12$ months following surgery and higher (up to $40 \%$ ) up to 5 years postoperatively. ${ }^{6}$ In our meta-analysis, we found that within 7.5 years of CABG, $31 \%$ of patients had developed dementia (when rigorous diagnostic methods were used) ${ }^{6}$ compared with the population prevalence of around $7 \%$ in Australasians aged $\geq 60$ years old. ${ }^{7}$

Delirium is also relevant to this patient group. It is a common form of postoperative cognitive dysfunction characterised by acute and fluctuating deficits in attention (or broader cognitive impairments) and arousal. ${ }^{8}$ Depending on cardiac surgery type, postoperative delirium incidence has been reported as high as $72 \%,{ }^{9}$ with our meta-analysis revealing the incidence after CABG was $24 \%$ when appropriate diagnostic tools were used. ${ }^{6}$ The presence of delirium in late life is associated with a doubling of cognitive decline,${ }^{10}$ leads to functional impairments, mortality ${ }^{11}$ and greatly increases the risk (OR 8.7) of dementia. ${ }^{11}$

Given CABG is such a common procedure (180476 in the USA in $2016^{12}$ ), reducing the incidence of postoperative cognitive impairment and delirium is a research priority. The worldwide cost of dementia in 2016 was estimated to be US $\$ 818$ billion and was projected to increase substantially in the following years. ${ }^{13}$ Therefore, it is crucial for the economy to reduce or delay cognitive impairments (including dementia) in late life, and targeting post-CABG cognitive dysfunction is a new promising strategy.

Prior research to prevent post-CABG cognitive impairment has met with little success, either through the alteration of surgical techniques, ${ }^{14}{ }^{15}$ or pharmacological and anaesthetic interventions. ${ }^{16-18}$ More advanced surgical methods such as increasing intraoperative systemic perfusion and hypothermia have shown some evidence of therapeutic efficacy but require expertise and technology not routinely available in hospitals. ${ }^{19}$ A novel approach is to consider that cognitive impairment in older adults comes about from the deterioration of multiple systems in a complex interplay. ${ }^{20}$ Interactions between general anaesthesia, cardiac output and reductions in mental abilities could be targeted by directly challenging cognitive systems. For example a recent study investigating the effect of postoperative computerised cognitive training (CCT) in 51 CABG patients found improvements in the trained domains of memory and attention $\left(\eta^{2}=0.50\right)$ at 3 months postoperatively in the intervention group, as compared with controls. ${ }^{21}$

CCT is an inexpensive, safe and scalable method of cognitive intervention which can be modified to target singular or multiple cognitive domains, and has been shown efficacious for cognition in older populations with and without cognitive impairments and in those with heart failure. ${ }^{22-26}$ A meta-analysis by Hill and colleagues ${ }^{22}$ reported a moderate effect size $(\mathrm{g}=0.35)$ on overall cognition in older adults with mild cognitive impairment. Healthy older adults and those with Parkinson's disease still benefit from CCT, although the overall pooled effect is not as large $(\mathrm{g}=0.22-0.23){ }^{22}{ }^{25} \mathrm{CCT}$ has also been shown effective in patients with heart failure, significantly improving processing speed. ${ }^{24}{ }^{26}$ The idea of optimising perioperative brain health, using cognitive training, is fast becoming recognised as an important area of clinical and public health research. ${ }^{27}$ Recently two studies investigating non-cardiac patients (lung transplant and knee arthroplasty) have shown improvement in neuropsychological testing ${ }^{28}{ }^{29}$ and reductions in depression and anxiety $^{29}$ following postoperative CCT.

To date, preoperative cognitive training has been used in three studies with both cardiac $^{30}$ and non-cardiac patients. ${ }^{31}{ }^{32}$ In gastrointestinal patients, a cognitive intervention delivered preoperatively decreased the incidence of acute (1 week) cognitive impairments postoperatively $(16 \%)$ when compared with the control group $(36 \%){ }^{31}$ However, no significant effects of presurgery cognitive training were found in feasibility trials investigating cardiac patients, ${ }^{30}$ nor a mixed group of gastrointestinal, urological, spine and hepatobiliary surgery patients, ${ }^{32}$ on either surgery-related cognitive impairment or delirium incidence. However, this may be due to the method of cognitive training administration, with each session being relatively short $(<30 \mathrm{~min})$ and home-based (unsupervised). Sessions of this format have been shown to be ineffective, especially if home-based training is not supplemented with supervised sessions. ${ }^{25}$

This project will deliver cognitive training preoperatively and postoperatively in older patients aged $\geq 65$ years undergoing CABG. Reducing postoperative cognitive impairments, including delirium, could have widereaching effects. Delirium is not only a risk factor of mortality but also prolongs admissions, which affect both the patient and the healthcare system financially. ${ }^{33} 34$ Additionally, better long-term cognitive outcomes may lead to increased healthcare access for older patients being considered for surgery, where they otherwise may have been declined due to cognitive concerns.

We aim to investigate the effect of remotely supervised CCT on cognitive outcomes following CABG surgery in older adults. Specifically, our primary aims are to determine the effect of (1) pre-CABG CCT on the incidence of postoperative delirium and (2) pre-CABG and postCABG CCT on cognitive decline (as compared with baseline) at 4 months. Our secondary aims are to determine the effect of (1) pre-CABG CCT on cognitive decline (as compared with baseline) at discharge and (2) pre-CABG and post-CABG CCT on cognitive decline (as compared with baseline) at 6 months and 1 year postsurgery. Additional exploratory aims are to determine the influence 


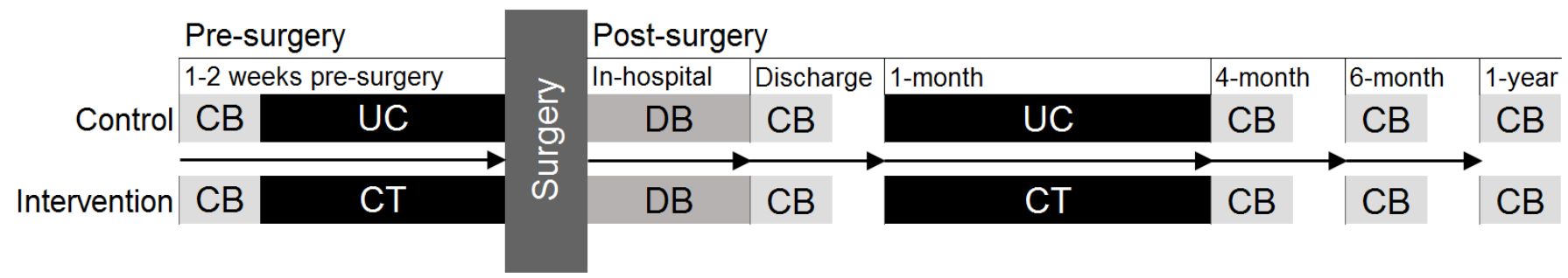

Figure 1 Study timeline highlighting the time of assessments and interventions, specific to control and intervention. Assessment sessions contain cognitive battery and other additional assessments (see table 1). CB, cognitive battery; CT, cognitive training; DB, daily delirium battery in hospital; UC, usual care.

of CCT on delirium severity and subtype, as well as the predictors of cognitive training response in older adults undergoing elective CABG surgery, including factors such as depression, education, age, baseline cognition and perioperative health.

\section{METHODS AND ANALYSIS \\ Study design}

This study is reported in alignment with the statements of the Consolidated Standards of Reporting Trials and the Standard Protocol Items: Recommendations for Interventional Trials. ${ }^{35} 36$ This study is a single-blinded, randomised controlled trial with longitudinal follow-up comparing remotely supervised CCT (intervention group) with usual care (control group). Cognitive function will be measured prior to surgery (baseline) and at discharge, 4 months (end of intervention), 6 months and 1 year postoperatively. The presence of delirium will be measured (daily) in-hospital postoperatively and at discharge (see figure 1).

\section{Patient and public involvement}

Clinical partners were involved in the study design; however, there was no patient and public involvement.

\section{Primary and secondary outcomes}

The effect of cognitive training will be assessed, through group comparison (control vs intervention), at multiple timepoints (as compared with baseline/pre-CABG, ie, change from baseline at multiple timepoints) throughout the study. Our primary outcome for cognitive decline (from baseline) is 4 months post-CABG, which follows cessation of the CCT intervention. The primary outcome for delirium will be presence (vs absence) in-hospital (including discharge assessment) post-CABG. Cognitive decline (from baseline) at discharge and at 6 months and 1 year post-CABG is also being investigated as secondary outcomes. Additional analyses will be performed to assess the exploratory aims of the study.

\section{Setting}

This research is ongoing and conducted at multiple sites (hospital, home, university). Recruitment and postCABG testing until discharge are conducted at the Royal Adelaide Hospital (RAH) in the Cardiothoracic Surgery
Unit and in the Intensive Care Unit. The RAH is a public hospital located in Adelaide central business district (CBD) providing services primarily to those in the central metropolitan region and a substantial number of people from rural and remote areas. Baseline and discharge testing are conducted at participants' homes. Follow-up testing sessions at 4 months, 6 months and 1 year postCABG are conducted at participants' homes or at a University of South Australia metropolitan campus.

\section{Participants}

Inclusion criteria

- Persons, male or female, who have consented to undergo elective CABG surgery at the RAH (note: additional concomitant cardiac surgeries will not exclude patient, such as CABG plus mitral valve replacement).

- Aged 65 years and over.

- Proficient English speakers.

- Have normal vision and hearing with or without the use of aids.

- Live within the metropolitan boundaries of Adelaide (Development Act 1993) or within a 1-hour drive from the CBD.

\section{Exclusion criteria}

- Known learning disabilities, a dementia diagnosis or a significant neurological/psychiatric diagnosis (all factors will affect ability to perform assessments).

- History of pharmaceutical cancer treatment within the past 5 years, excluding pure surgical treatment (cancer treatment is known to negatively affect $\operatorname{cognition}^{37}$ ).

- Suffered a stroke within the past year (many strokerelated cognitive impairments subside within a year ${ }^{38}$ ).

- Currently practising cognitive training (will interfere or muddle cognitive training effects).

- Additionally, those who are not enrolled to complete baseline testing at least 5-6 days prior to their surgery will be excluded (need to complete a minimum of three preoperative cognitive training sessions).

Sample size

Using the $G^{*}$ Power statistical analysis software, ${ }^{39}$ a priori sample size was calculated based on a published 
meta-analysis effect size (Hedges' $\mathrm{g}=0.35$ ) of the overall effect of CCT on cognitive outcomes (in adults aged $\geq 60$ years who presented with mild cognitive impairment). ${ }^{22}$ The selection of this effect size for cognitive training (previously mentioned meta-analysis) was based on the cognitive status distribution of those already recruited within our study, with over half presenting with cognitive impairment at baseline (Addenbrooke's Cognitive Examination (ACE-III) score $\leq 88$ ). This power analysis is based on the primary study outcomes: presence of cognitive decline at 4-month follow-up and presence of delirium following surgery. With $g=0.35$, power $=0.80$ and $\alpha=0.05$, the computed sufficient sample size for comparing presence versus absence of delirium or cognitive decline using logistic regression was $\mathrm{n}=94$ ( 47 per group); therefore, in order to account for participant attrition during follow-up, a total of 120 participants (60 per group) will be recruited.

\section{Recruitment and feasibility}

Patients are recruited during their presurgery clinic day at the RAH, approximately $1-3$ weeks prior to CABG surgery. Presurgery clinic days are designed to educate the patient about their upcoming surgery and the possible outcomes of the surgery, and allows nursing, pharmacy, medical and anaesthetic staff to meet with the patients and gain any further necessary information (lung function, height, weight and ECG). Patients are informed of the study face to face (or via a phone call if not seen during the presurgery clinic) during the afternoon session; those interested are then screened for inclusion and if eligible and interested are scheduled for their baseline session (informed consent process is conducted during baseline session). As recruitment is under way, the current rate is approximately 25 participants a year (27 participants from May 2018 to July 2019). To ensure numbers are met, active recruitment will be conducted for approximately 4.5 years in total.

\section{Honoraria}

Participants will receive a total of $\$ 100(5 \times \$ 20)$ worth of vouchers across the duration of the study. The initial voucher is supplied to participants after the conclusion of their baseline testing session, prior to their surgery. The remaining four vouchers will be supplied to participants after completion of the discharge and at 4-month, 6-month and 1-year post-CABG follow-up sessions. Honoraria are being used in this study to cover time and other costs associated with participation.

\section{Measures}

Cognitive battery

Cognition is assessed with the ACE-III and multiple tests from the Cambridge Neuropsychological Test Automated Battery (CANTAB). The ACE-III is a widely used global cognition examination designed to screen for dementia. It assesses attention, language, verbal memory and visuospatial function and is scored out of 100 (higher scores indicate better cognition). The two clinical cut-offs show high sensitivity and specificity: mild cognitive impairment 88 (sensitivity $=1.0$; specificity $=0.96$ ) and dementia 82 (sensitivity $=0.93$; specificity $=1.0$ ). ${ }^{40}$ Additionally, the ACE-III has alternate versions (all used within the study), allowing it to be used for longitudinal testing. The ACEIII score will be used to characterise and provide clinical meaning, at all study timepoints, categorising participants as normal, mild cognitive impairment or dementia according to clinical score cut-offs.

The CANTAB connect system provides a portable neuropsychological testing battery which can detect changes in multiple cognitive domains. The CANTAB testing battery used focuses on cognitive domains we know to be affected most by coronary artery disease, including psychomotor speed, memory and executive function. ${ }^{3}$ This allows us to detect smaller changes in specific cognitive domains, unlike the global cognition score from the ACE-III. Additionally, the CANTAB system contains parallel versions of tests which minimise practice effects of the tasks within longitudinal studies. The specific tests used will be the Motor Orientation Task (MOT), Reaction Time (RTI), Paired Associated Learning (PAL), Spatial Working Memory (SWM) and One Touch Stockings of Cambridge (OTS). Data from CANTAB will be converted into one main outcome measure for each test and used to investigate cognitive decline (over time). Data from the MOT will not be analysed as this test is purely a tablet familiarisation task. It should be noted that in the initial cognitive assessment during baseline testing, CANTAB is conducted twice (practice trials approximately $15 \mathrm{~min}$, then full assessment including practice trials), serving as a double baseline in an attempt to minimise practice effects of the tasks.

\section{Delirium battery}

The presence of delirium (primary aim) and the severity and motor subtype (characterisation and exploratory purposes) are captured with the Confusion Assessment Method (CAM) and CAM for the Intensive Care Unit (CAM-ICU) version and the Memorial Delirium Assessment Scale (MDAS). Following surgery, while patients are in the intensive care unit, the CAM-ICU is used to diagnose the presence of delirium. The CAM-ICU is an adaptation of the original CAM which has been validated and has high sensitivity and specificity in those who are critically ill or those who receive multiple psychoactive medications (ie, postoperative patients). ${ }^{41}$ While on the ward a daily MDAS interview is conducted to ascertain delirium severity, as the MDAS is not intended for diagnostic purposes. ${ }^{42}$ This interview informs the scoring of the CAM (questions used to score the CAM are embedded in the MDAS interview), providing a dichotomous delirium presence outcome. On weekends, when delirium assessments are not conducted by research team members, a chart-based review tool developed by Inouye and colleagues ${ }^{44}$ is used to ascertain the presence of any delirium episodes. We acknowledge that the use of a 
chart review tool is not as thorough as our daily assessment battery and may result in inaccuracies (likely underestimation) in determining delirium.

The CAM is divided into four main features of delirium: (1) acute onset and fluctuating course; (2) inattention; (3) disorganised thinking; and (4) altered level of consciousness. To assess altered level of consciousness/ arousal, two scales were selected. The Richmond Agitation Sedation Scale is used in the ICU, while the Observational Scale of Level of Arousal is used on the ward. The primary outcome (presence vs absence of delirium) is considered as positive delirium, both in the ICU and during the ward stay, when CAM features 1 and 2 and either 3 or 4 are present. A positive delirium diagnosis, or acknowledgement of delirium in chart review, at any point during the hospital stay is considered as presence of delirium. All members conducting delirium assessments were trained by qualified geriatricians $(\mathrm{AB}$ and $\mathrm{DHJD})$.

For characterisation of delirium and for exploratory purposes of cognitive training effects, the severity and subtype of delirium are also assessed. The MDAS interview contains a cognitive assessment which allows delirium items of memory impairment, impaired digit span and inattention to be easily quantified, as well as more open-ended questions regarding patients' experiences to assess sleep-wake cycle, perceptual disturbance and disorganised thinking. This allows a severity score out of 30 (higher scores indicate more severe symptoms) to be generated. Motor subtype (hypoactive, hyperactive, mixed or no subtype) of delirium, when present, is classified using a checklist created by Meagher. ${ }^{45}$

\section{Computerised cognitive training}

Given cognitive training gains tend to be most profound in trained domains, the CCT intervention used in this trial is based on HappyNeuron Pro, which provides extensive practice on the cognitive domains most affected by heart failure, namely psychomotor speed, attention, memory and executive function. ${ }^{24} 4647$ In addition, this program offers a clinical backend, which provides remote monitoring of performance and adherence and thus facilitates remote supervision aimed at maximising participant engagement.

For participants in the intervention group, the CCT intervention is home-based and conducted on either a participant's personal laptop or on a supplied universityowned laptop and Wi-Fi dongle. The duration of the sessions is approximately $45-60 \mathrm{~min}$ of completed cognitive training (allowing for short breaks). The session frequency pre-CABG is every other day (3.5 times a week on average), while post-CABG this frequency decreases to three times a week (see figure 1). This dosage has been established as the most effective in a meta-analysis, where sessions less than $30 \mathrm{~min}$ in length or more than three sessions a week were found less efficacious than longer, less frequent sessions. ${ }^{25}$

Pre-CABG cognitive training begins following baseline testing, conducted at the participant's home, commonly
1-2 weeks prior to scheduled CABG. The initial session (during baseline) and the following session are instructed and supervised, with an emphasis being placed on participants' understanding, strategy and competence of system use. Participants complete approximately $45-60 \mathrm{~min}$ of cognitive training every other day over a 2-week period following baseline testing (minimum of three sessions). All participants are supervised for at least one session a week as well as contacted weekly via phone; these calls are in place to help participants with any technical problems and to assess their weekly pain score.

Post-CABG cognitive training begins 1 month following surgery, allowing enough recovery time (timing decided after consultation with study clinical partners). This second regimen is longer in duration (compared with pre-CABG) and is conducted for 12 weeks, still with 45-60 min sessions three times a week (approximately 27-30 hours). Between their pre-CABG and post-CABG interventions, participants are advised not to access the CCT program. The initial session is yet again supervised; following this, one session per week is supervised. Weekly follow-up phone calls are conducted identical to the pre-CABG intervention. Following the completion of the intervention, participants will be given access to the cognitive training system if they request it until the completion of the study (1 year postoperatively); however, there will be no scheduled sessions.

When required (due to participant struggles or intervention inactivity), an additional face-to-face supervised session is organised. However, when inactivity or a lack of adherence is common during the post-CABG intervention, participant involvement in the intervention is ceased. An acceptable adherence cut-off has been set as completion of $66 \%$ (standard within the literature) of prescribed training sessions. ${ }^{48}$ If a participant is not reaching this target, considering holidays (Christmas/Easter) and any relevant personal reasons, they are notified of the consequences of not improving their adherence to the accepted rate. If the lack of adherence continues, they are terminated from the intervention but offered to stay enrolled in the study for follow-up (however, their data will not be used in group comparisons).

Participants randomised to the control group experience usual care, with no intervention both pre-CABG and post-CABG. However, these participants are still contacted via phone on a weekly basis to complete their pain assessments.

\section{Procedure}

Participants are randomised into either control or intervention and provide informed consent prior to the commencement of the first data collection session (baseline). Approximately 1-2 weeks prior to their surgery (minimum 5-6 days), baseline testing (up to 3-4hours) is conducted, including demographics, psychological (depression), general questionnaires (health, well-being, quality of life), pain measures and cognitive assessment, 
along with the commencement of their intervention programme, if in the intervention group (see table 1).

Information regarding perioperative factors are recorded, including anaesthetics, time on bypass and aortic cross-clamp time. Following surgery, clinical data (bedside notes, medicine charts) and delirium episodes are monitored daily until discharge by a researcher. Following discharge (within 7 days of leaving the RAH), a cognitive assessment identical to baseline is conducted by a researcher who is blind to group allocation, at the participant's home (discharge testing). A home visit was decided as the most practical and feasible method of collecting discharge data after consultation with clinical partners involved in the study. Finally, cognitive assessments are performed during follow-up sessions at approximately 4 months (on postoperative intervention programme completion), 6 months and 1 year postCABG, at a University of South Australia metropolitan campus or the participant's home.

\section{Randomisation}

Once a potential participant has confirmed their interest, they are booked in for their baseline session. Once scheduled, they are allocated a study identification (ID) number (next sequential number after the previous participant) and randomised into either control or intervention (50:50) based on this ID number. A randomisation list was generated (www.randomization.com) prior to study commencement, with specification ensuring that for every 10 participants, 5 will be randomised to each group. This list is only accessible by one member of the research team, who only informs research team members of an individual participant's group allocation when needed.

\section{Blinding}

The blinding procedure within this study is complex, as multiple research team members are involved in baseline sessions and providing face-to-face cognitive training sessions to participants. At baseline, two research team members are always present for the session, not only for safety but also to ensure neuropsychological tests are administered by a blinded (to group allocation) research team member. One of the team members present at baseline, who is not blinded, conducts the consent process for the participant, while the other researcher is not present. The other team member, who is blinded, returns following consent and administers the cognitive test battery. This team member by the conclusion of the baseline session is no longer blinded, as the participant will either complete a cognitive training session at the end of their baseline testing or not. Delirium assessments in hospital are always conducted by a researcher who is blinded to the group allocation of the participant (this researcher never attends baseline sessions). Additionally, all discharge and follow-up assessments are conducted by a research team member who is blinded to group allocation. In this study, separate consent forms are used for each group (different consent forms for those in control vs intervention). Therefore, the participants are blinded to the presence of other study groups.

\section{Data analysis}

To determine the cognitive effects of CCT on delirium incidence and cognitive decline post-CABG, a per-protocol analysis method will be employed, where only data from participants who completed $\geq 66 \%$ of prescribed CCT will be included in the primary analyses (not applicable to the control group). ${ }^{48}$ It is known that using this approach can bring about problems such as sample biases, reduced sample size and limited generalisability. ${ }^{49}$ Yet, due to this study being the first of its kind, it is important to determine the efficacy of the intervention as described, which will provide a platform for future and larger research projects (which could incorporate intention-to-treat approaches). ${ }^{49}$ Additionally, due to the non-linear nature of cognitive function from pre-CABG to post-CABG, no missing data approaches (eg, carry one forward or growth curve) would be suitable. This non-linearity is evident in our recent meta-analysis, where cognitive impairment is present in $19 \%$ of CABG patients preoperatively, then postoperatively increases to $43 \%$ acutely, improves to $19 \%$ at 4-6 months, then deteriorates to $40 \%$ at $1-5$ years. ${ }^{6}$ For our primary analyses (delirium: delirium presence; cognitive decline: 4 months), we will therefore only include participants without missing data. For all analyses, effect sizes will be reported and discussed.

\section{Primary and secondary outcomes}

\section{Delirium}

Delirium (presence vs absence across hospital stay) will be the outcome variable in a logistic regression, with group membership (control vs intervention) as a predictor variable, and age and gender as covariates. Sensitivity analyses will be run with additional covariates, including baseline cognition (ACE-III score) and depression, to ensure any effects are not due to residual confounding, as these factors are related to delirium incidence.

\section{Cognitive decline}

Cognitive decline will be calculated using the Reliable Change Index (RCI) method, across the four CANTAB tests (main outcome measure will be used for RTI, PAL, SWM and OTS). This method was recommended to be used for future research investigating cognitive decline in our recent meta-analysis. ${ }^{6}$ The RCI method reflects individual change between two timepoints (from baseline to follow-up timepoint), relative to the whole sample data while considering practice effects of the test. Specifically, for each main test outcome, for each of the four CANTAB tests (RTI, PAL, SWM and OTS), the change in the participant's pre-CABG to post-CABG test score is divided by the SE of the difference between the two test scores. Significant cognitive decline of an individual will be considered if a score of $\geq-1.64$ in $\geq 1$ of the four tests is present, resulting in a dichotomous variable for each 


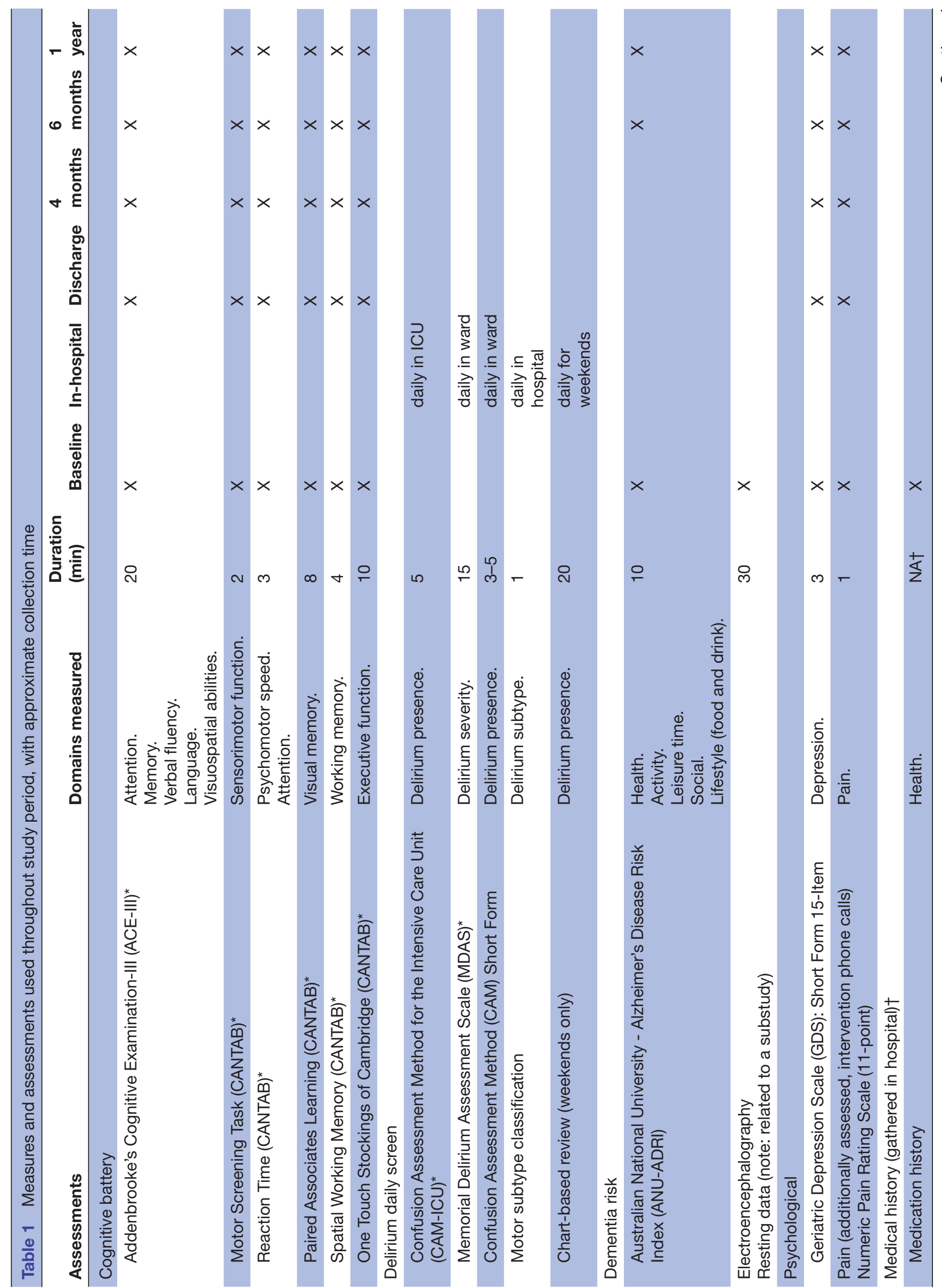

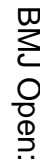

$\stackrel{\vec{F}}{\stackrel{9}{9}}$

음

$\frac{\overline{0}}{\overline{0}}$

क

$\vec{\circ}$

$\overrightarrow{\vec{\omega}}$

홍.

D

옹

它

ज़

운

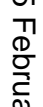

ฏ

옹

ํํำ

흥

을. 


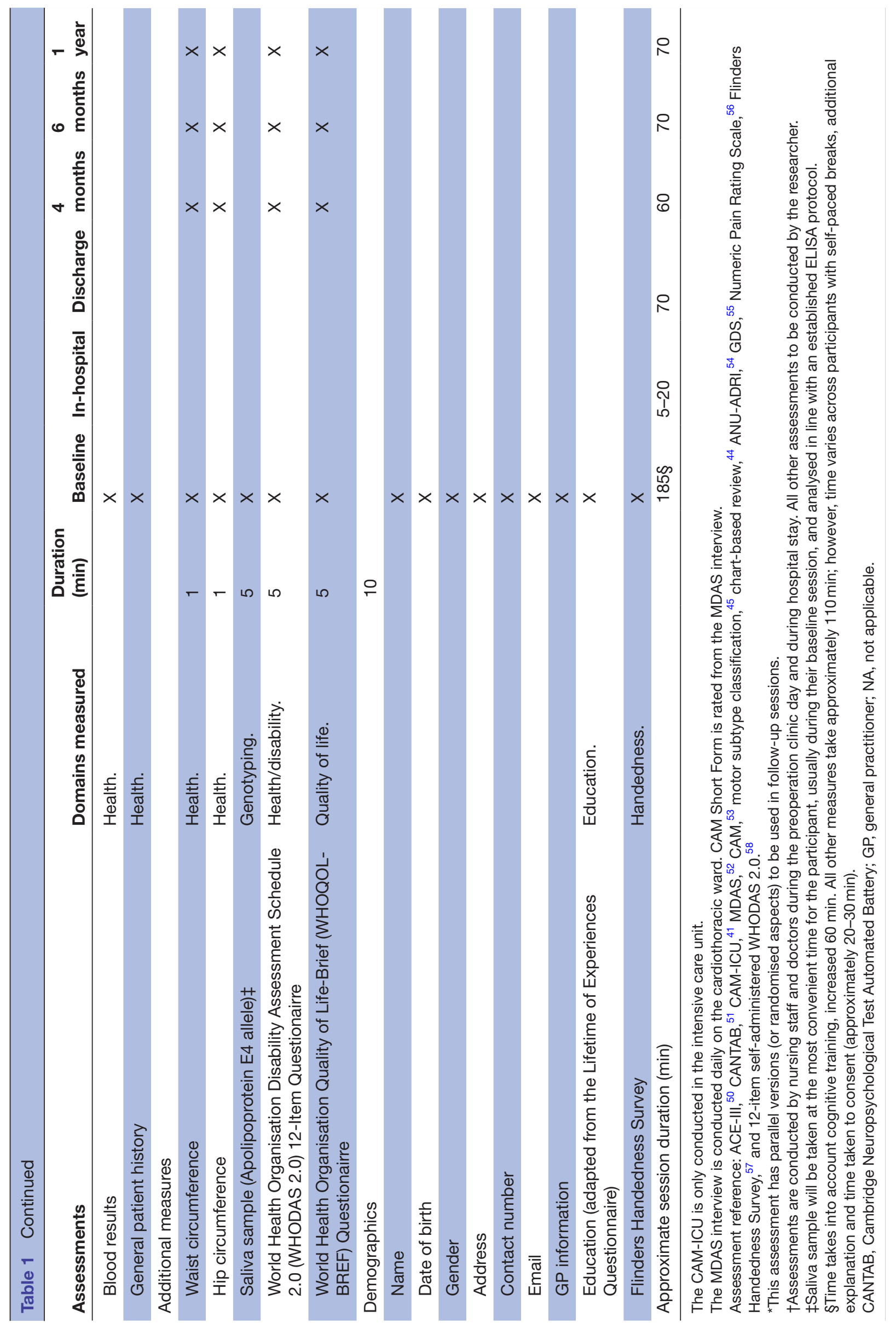

س

$\frac{0}{8}$

$\stackrel{\vec{\nabla}}{\stackrel{9}{+}}$

응

$\frac{\text { क्र }}{\bar{D}}$

\%

。

$\overrightarrow{\vec{\omega}}$

$\stackrel{5}{3}$.

욤

$\stackrel{+}{\circ}$

它 
participant (decline vs no decline/improvement). For the primary cognitive outcome at 4 months post-CABG, cognitive decline (decline vs no decline/improved) will be analysed by logistic regression with group membership (control vs intervention) as the predictor variable, and covariates of age and gender. Sensitivity analyses will be run with additional covariates including baseline cognition (ACE-III score) and depression, to ensure any effects are not due to residual confounding, as these factors are related to cognitive decline. For the secondary outcomes of all other follow-up sessions (discharge, 6 months and 1 year), cognitive decline at each timepoint (always compared with pre-CABG cognition) will be used in separate logistic regressions, with group membership (control vs intervention) as the predictor variable, and covariates of age and gender.

\section{Exploratory delirium analyses}

Additional delirium outcomes (other than presence vs absence) will be assessed, including severity, duration of episode and motor subtype, with the same predictors described in previous analyses. Delirium severity (MDAS score) and days spent in a delirium episode will be investigated using linear regression, and delirium motor subtype (hypoactive/hyperactive/mixed) will be investigated using multinominal logistic regression. These analyses will be conducted to investigate whether cognitive training affects delirium characteristics (as opposed to presence).

\section{Exploratory cognitive decline analyses}

To identify significant predictors of response to cognitive training, a series of univariate linear regressions will be conducted with the outcome being cognition change (RCI scores, as a continuous variable, calculated using CANTAB data for each participant at each timepoint), and predictor variables will include baseline dementia risk, apolipoprotein E4 (APOE4) genotype, depression, pain, delirium, gender, age, cardiovascular risk (cholesterol, glucose, blood pressure), medical history, quality of life and disability, body mass index, waist to hip ratio, waist circumference, medications, education, baseline cognition (ACE-III score), and perioperative factors (anaesthetics, time on bypass, time aorta clamped, number of bypasses, surgical complications). These analyses will be conducted on data obtained at discharge and at 4-month, 6-month and 1-year follow-up separately.

Additionally, to investigate the presence of a dose response regarding CCT, a univariate linear regression will be conducted with the outcome being cognitive change (individual participant RCI scores, described previously) and the predictor variable of CCT training dose (total CCT minutes). Separate analyses will be conducted for (1) preoperative CCT training dose as compared with discharge cognitive change data, and (2) postoperative CCT training dose as compared with 4-month, 6-month and 1-year follow-up data separately.
The cognitive outcomes investigated in these analyses will be conducted on the data of participants who meet the standardised adherence cut-off of $66 \%$. It should be noted that these additional analyses are exploratory and aim to establish effect sizes for future studies and will only be conducted on data obtained from the intervention group.

\section{ETHICS AND DISSEMINATION Informed consent}

Participants interested and eligible are provided with both written and verbal information regarding the study by a study researcher (see online supplementary consent forms for control and intervention participants). The participants are reassured that the decision to participate or not will not influence the care provided to them at the RAH. Participants who present with a clinical diagnosis of dementia or learning disability are not eligible for the study, and therefore all potential participants should have the ability to give informed consent.

All participants reconsent at the 6-month and 1-year follow-up sessions. Again, participants are provided with both written and verbal information regarding the study by a researcher. If participants, since the last consent process, have been clinically diagnosed with dementia, or have developed a disability leaving them unable to consent for themselves, then a person responsible for the participant provides the written informed consent. The information is still discussed with both parties, and if the participant indicates that they would not like to participate further then they are withdrawn from the study. Additionally, all participants are free to withdraw from the study at any time with no consequences to them now or in the future.

\section{Data management}

All data obtained during the study, including data from hospital records (hard copy hospital records do not leave hospital), are entered into a password-protected electronic database (REDCap). Data are kept in a locked cabinet (hard copy) and stored on a password-protected server (electronic). The research team has access to hard copy data, and passwords are only known by members of the team who enter and analyse data. Data will be stored for a minimum of 5 years from study completion. A data monitoring committee is not used within this study as CCT interventions have no known risks. Any interim analyses will be conducted by researchers not directly involved with the participants.

\section{Saliva sample collection}

A saliva sample is collected from participants during the study to investigate the APOE4 allele genetic marker of cognitive impairment. The collection of saliva is noninvasive and poses no risk to the participant as drool is collected in an Oragene kit. This sample is taken at the most convenient time for the participant, usually during 
their baseline session. It should be noted that only participants who consent (tick the box in relation to the explicitly stated genetic marker testing on the consent form) will have genetic data extracted from their sample. The genetic data will be de-identified (reidentifiable) and stored in a password-protected file. Only a member of the research team or the research lab technician employed to analyse the sample has access to the tissue sample itself after data collection.

\section{Risks}

The proposed intervention programme poses no additional risks to the participants as it does not interfere with their surgery or their cardiac rehabilitation. There have additionally been no risks of cognitive training interventions noted in the literature. No data collection methods such as saliva, cognitive testing and electroencephalography (which is part of a substudy not described here) pose any risks to participants.

There are small risks associated with home-based data collection for researchers; however, methods to lower this risk have been put in place. An initial hazard risk screening is conducted with the participant regarding their home and any possible risks (ie, dogs, housemates, stairs and so on). A log-in safety system is also used online where if the researcher fails to check in after a designated time (ie, 4hours for baseline session), an emergency message is sent out to nominated contacts.

\section{Ethical considerations}

In the case that a participant scores within clinical ranges for any tests, a letter is sent to their general practitioner notifying them of the study and their result (if not within hospital), or the appropriate staff within the hospital are notified (eg, home treating team for delirious patients).

\section{Dissemination}

It is anticipated that the results of this study will inform multiple publications and will be presented at multiple national and international conferences. As this is a topical area of research, results will be published throughout multiple stages of the study period and succeeding follow-ups, to ensure findings are communicated quickly and directly.

\section{Author affiliations}

${ }^{1}$ Cognitive Ageing and Impairment Neurosciences Laboratory (CAIN), School of Psychology, Social Work and Social Policy, University of South Australia Division of Education, Arts and Social Sciences, Adelaide, South Australia, Australia

${ }^{2}$ Adelaide Medical School, The University of Adelaide, Adelaide, South Australia, Australia

${ }^{3}$ Vascular Research Centre, South Australian Health and Medical Research Institute, Adelaide, South Australia, Australia

${ }^{4}$ Department of Cardiology, Royal Adelaide Hospital, Central Adelaide Local Health Network, Adelaide, South Australia, Australia

${ }^{5}$ Academic Unit for Psychiatry of Old Age, Department of Psychiatry, The University of Melbourne, Melbourne, Victoria, Australia

${ }^{6}$ Department of Neurology, Charité Universitätsmedizin Berlin, Berlin, Germany ${ }^{7}$ MRC Unit for Lifelong Health and Ageing, University College London, London, UK

${ }^{8}$ Alliance for Research in Exercise, Nutrition and Activity (ARENA), University of South Australia Division of Health Sciences, Adelaide, South Australia, Australia
${ }^{9}$ Department of Geriatric and Rehabilitation Medicine, Royal Adelaide Hospital, Central Adelaide Local Health Network, Adelaide, South Australia, Australia ${ }^{10}$ Department of Cardiothoracic Surgery, Royal Adelaide Hospital, Central Adelaide Local Health Network, Adelaide, South Australia, Australia

${ }^{11}$ Brain and Mind Centre and Sydney Medical School, The University of Sydney, Sydney, New South Wales, Australia

Acknowledgements The authors would like to acknowledge Erica Ghezzi for creating the figure used in the manuscript, and all research assistants, honour students and PhD candidates involved with data collection. Additionally, the authors would like to extend their thanks to HappyNeuron Pro for allowing access to their cognitive training system used within this intervention.

Contributors DG, HADK, PJP and DHJD conceived the paper. DG, HADK, PJP, AL, DHJD, AES and MJV were involved in the development of the intervention and outcome measurements. DHJD, PJP, AB and MGW are clinical experts and provided input into all clinical aspects, including recruitment strategy. DG drafted the manuscript, and all authors revised and approved the final manuscript.

Funding DG is supported by the Australian Government Research Training Program Scholarship. HADK is supported by an NHMRC Boosting Dementia Research Leadership Fellowship (GNT1135676) and the National Heart Foundation of Australia Vanguard Grant (101758 - VG 2017). PJP is supported by a National Heart Foundation of Australia Future Leader Fellowship (FLF100412) and an NHMRC Career Development Fellowship (CDF1161506). AL is supported by an NHMRC-ARC Dementia Research Development Fellowship (GNT1108520). DHJD is supported by a Wellcome Trust Intermediate Clinical Fellowship (WT107467). AES is supported by an NHMRC-ARC Dementia Research Development Fellowship (GNT1097397). MJV is supported by an NHMRC Career Development Fellowship (GNT1112813). This study is supported by NMHRC funding from HADK.

Competing interests The computerised intervention is provided for the study by HappyNeuron Pro at no cost to the research team. However, HappyNeuron Pro has no input as to the dissemination of the results of this study. AL codeveloped systems for remote delivery of computerised cognitive training as part of industry collaborations with Synaptikon funded by the Australian National Health and Medical Research Council (NHMRC GNT1095097) and the German Federal Ministry of Education and Research (BMBF grant 13GW0212A), but has no financial interests in these or any other computerised cognitive training program. MJV is scientific founder of the University of Sydney spin-out company Skin2Neuron, where he has a financial interest unrelated to this work. He receives inkind research support from NeuroNation (Synaptikon) and COGSTATE towards the National Health and Medical Research Council of Australia-funded Maintain Your Brain Dementia Prevention Trial.

Patient consent for publication Not required.

Ethics approval The ethical aspects of this research have been approved by the HREC of Royal Adelaide Hospital (reference number: HREC/17/RAH/445), Central Adelaide Local Health Network (reference number: R20171020) and the University of South Australia Human Ethics Committee (reference number: 0000034053). Original approval was received on 13 December 2017. This study is subject to standard institutional and ethical auditing processes.

Provenance and peer review Not commissioned; externally peer reviewed.

Open access This is an open access article distributed in accordance with the Creative Commons Attribution Non Commercial (CC BY-NC 4.0) license, which permits others to distribute, remix, adapt, build upon this work non-commercially, and license their derivative works on different terms, provided the original work is properly cited, appropriate credit is given, any changes made indicated, and the use is non-commercial. See: http://creativecommons.org/licenses/by-nc/4.0/.

ORCID iD

Danielle Greaves http://orcid.org/0000-0002-2594-4492

\section{REFERENCES}

1 Alexander JH, Smith PK, Grafting C-AB. Coronary-Artery bypass grafting. N Engl J Med Overseas Ed 2016;374:1954-64.

2 McKhann GM, Grega MA, Borowicz LM, et al. Is there cognitive decline 1 year after CABG?: comparison with surgical and nonsurgical controls. Neurology 2005;65:991-9.

3 Vogels RLC, Scheltens P, Schroeder-Tanka JM, et al. Cognitive impairment in heart failure: a systematic review of the literature. Eur $J$ Heart Fail 2007;9:440-9. 
4 Dardiotis E, Giamouzis G, Mastrogiannis D, et al. Cognitive impairment in heart failure. Cardiol Res Pract 2012;2012:1-9.

5 Velazquez EJ, Lee KL, Jones RH, et al. Coronary-Artery bypass surgery in patients with ischemic cardiomyopathy. N Engl J Med 2016;374:1511-20.

6 Greaves D, Psaltis PJ, Ross TJ, et al. Cognitive outcomes following coronary artery bypass grafting: a systematic review and metaanalysis of 91,829 patients. Int J Cardiol 2019;289:43-9.

7 Prince M, Bryce R, Albanese E, et al. The global prevalence of dementia: a systematic review and metaanalysis. Alzheimer's \& Dementia 2013;9:63-75

8 Fong T, Davis D, Growdon M, et al. The interface of delirium and dementia in older persons. Lancet Neurol 2015;14:823-32.

9 Sockalingam S, Parekh N, Bogoch II, et al. Delirium in the postoperative cardiac patient: a review. J Card Surg 2005;20:560-7.

10 Fong T, Inouye S, Delirium JR. Dementia, and decline. JAMA Psychiatry 2017;74:212-3.

11 Davis DHJ, Muniz Terrera G, Keage H, et al. Delirium is a strong risk factor for dementia in the oldest-old: a population-based cohort study. Brain 2012;135:2809-16.

12 D'Agostino RS, Jacobs JP, Badhwar V, et al. The Society of thoracic surgeons adult cardiac surgery database: 2018 update on outcomes and quality. Ann Thorac Surg 2018;105:15-23.

13 Prince M, Comas-Herrera A, Knapp M. World Alzheimer report 2016: improving healthcare for people living with dementia: coverage quality and costs now and in the future, 2016.

14 Sun J, Wu X, Wang W, et al. Cognitive dysfunction after off-pump versus on-pump coronary artery bypass surgery: a meta-analysis. $J$ Int Med Res 2012;40:852-8.

15 Kennedy ED, Choy KCC, Alston RP, et al. Cognitive outcome after on- and off-pump coronary artery bypass grafting surgery: a systematic review and meta-analysis. J Cardiothorac Vasc Anesth 2013;27:253-65.

16 Holinski S, Claus B, Alaaraj N, et al. Cerebroprotective effect of piracetam in patients undergoing coronary bypass burgery. Med Sci Monit 2008;14:Pi53-7.

17 Ottens TH, Dieleman JM, Sauër A-MC, et al. Effects of dexamethasone on cognitive decline after cardiac surgery: a randomized clinical trial. Anesthesiology 2014;121:492-500.

18 Waegemans T, Wilsher CR, Danniau A, et al. Clinical efficacy of piracetam in cognitive impairment: a meta-analysis. Dement Geriatr Cogn Disord 2002;13:217-24.

19 Bhamidipati D, Goldhammer JE, Sperling MR, et al. Cognitive outcomes after coronary artery bypass grafting. $J$ Cardiothorac Vasc Anesth 2017:31:707-18.

20 Gorelick PB, Scuteri A, Black SE, et al. Vascular contributions to cognitive impairment and dementia. Stroke 2011:42:2672-713.

21 de Tournay-Jetté E, Dupuis G, Denault A, et al. The benefits of cognitive training after a coronary artery bypass graft surgery. $J$ Behav Med 2012;35:557-68.

22 Hill NTM, Mowszowski L, Naismith SL, et al. Computerized cognitive training in older adults with mild cognitive impairment or dementia: a systematic review and meta-analysis. AJP 2017;174:329-40.

23 Kelly ME, Loughrey D, Lawlor BA, et al. The impact of cognitive training and mental stimulation on cognitive and everyday functioning of healthy older adults: a systematic review and meta-analysis. Ageing Res Rev 2014;15:28-43.

24 Ellis ML, Edwards JD, Peterson L, et al. Effects of cognitive speed of processing training among older adults with heart failure. J Aging Health 2014;26:600-15.

25 Lampit A, Hallock H, Valenzuela M. Computerized cognitive training in cognitively healthy older adults: a systematic review and metaanalysis of effect modifiers. PLoS Med 2014;11:e1001756.

26 Kua ZJ, Valenzuela M, Dong Y. Can computerized cognitive training improve cognition in patients with heart failure? J Cardiovasc Nurs 2019;34:E19-27.

27 Lenze EJ, Bowie CR, Avidan MS. Cognitive training for optimizing perioperative brain health. Am J Geriatr Psychiatry 2019;27:1228-31.

28 Song $Y$, Cui X, Zhang Y, et al. Home-Based computerized cognitive training for postoperative cognitive dysfunction after lung transplantation in elderly population. $J$ Nerv Ment Dis 2019;207:693-9.

29 Carbone E, Vianello E, Carretti B, et al. Working memory training for older adults after major surgery: benefits to cognitive and emotional functioning. Am J Geriatr Psychiatry 2019;27:1219-27.

30 O'Gara B, Mueller A, Gasangwa D, et al. Prevention of early postoperative decline: a randomized, controlled feasibility trial of perioperative cognitive training. Anesth Analg.

31 Saleh AJ, Tang G-X, Hadi SM, et al. Preoperative cognitive intervention reduces cognitive dysfunction in elderly patients after gastrointestinal surgery: a randomized controlled trial. Med Sci Monit 2015;21:798-805.

32 Vlisides PE, Das AR, Thompson AM, et al. Home-Based cognitive prehabilitation in older surgical patients. J Neurosurg Anesthesiol 2019;31:212-7.

33 Inouye SK, Westendorp RGJ, Saczynski JS. Delirium in elderly people. Lancet 2014;383:911-22.

34 Saravay SM, Kaplowitz M, Kurek J, et al. How do delirium and dementia increase length of stay of elderly general medical inpatients? Psychosomatics 2004;45:235-42.

35 Schulz K, Altman D, Moher D. Consort 2010 statement: updated guidelines for reporting parallel group randomised trials. $B M J ; 2010: c 332$.

36 Chan A-W, Tetzlaff JM, Altman DG, et al. Spirit 2013 statement: defining standard protocol items for clinical trials. Ann Intern Med 2013;158:200-7

37 Ahles TA, Root JC, Ryan EL. Cancer- and cancer TreatmentAssociated cognitive change: an update on the state of the science. JCO 2012;30:3675-86.

38 Park J-Y, Kim Y-H, Chang WH, et al. Significance of longitudinal changes in the Default-mode network for cognitive recovery after stroke. Eur J Neurosci 2014;40:2715-22.

39 Faul F, Erdfelder E, Lang A-G, et al. G*Power 3: a flexible statistical power analysis program for the social, behavioral, and biomedical sciences. Behav Res Methods 2007;39:175-91.

40 Noone P. Addenbrooke's Cognitive Examination-III. Occup Med 2015;65:418-20.

41 Ely EW, Margolin R, Francis J, et al. Evaluation of delirium in critically ill patients: validation of the confusion assessment method for the intensive care unit (CAM-ICU). Crit Care Med 2001;29:1370-9.

42 Gaudreau J-D, Gagnon P, Harel F, et al. Fast, systematic, and continuous delirium assessment in hospitalized patients: the nursing delirium screening scale. J Pain Symptom Manage 2005;29:368-75.

43 De J, Wand APF. Delirium screening: a systematic review of delirium screening tools in hospitalized patients. Gerontologist 2015;55:1079-99.

44 Inouye SK, Leo-Summers L, Zhang Y, et al. A Chart-Based method for identification of delirium: validation compared with Interviewer ratings using the confusion assessment method. J Am Geriatr Soc 2005;53:312-8.

45 Meagher D. Motor subtypes of delirium: past, present and future. Int Rev Psychiatry 2009;21:59-73.

46 Pressler SJ. Cognitive functioning and chronic heart failure: a review of the literature (2002-July 2007). J Cardiovasc Nurs 2008:23:239-49.

47 Vogels RLC, Oosterman JM, Van Harten B, et al. Profile of cognitive impairment in chronic heart failure. J Am Geriatr Soc 2007:55:1764-70.

48 Coley N, Ngandu T, Lehtisalo J, et al. Adherence to multidomain interventions for dementia prevention: data from the finger and MAPT trials. Alzheimers Dement 2019;15:729-41.

49 Ranganathan P, Pramesh CS, Aggarwal R. Common pitfalls in statistical analysis: intention-to-treat versus per-protocol analysis Perspect Clin Res 2016;7:144.

50 Hsieh S, Schubert S, Hoon C, et al. Validation of the Addenbrooke's cognitive examination III in frontotemporal dementia and Alzheimer's disease. Dement Geriatr Cogn Disord 2013;36:242-50.

51 Cambridge Cognition. Cantab connect. Cambridge, UK: Cambridge Cognition Ltd.

52 Breitbart W, Rosenfeld B, Roth A, et al. The Memorial delirium assessment scale. J Pain Symptom Manage 1997:13:128-37.

53 Inouye S, van Dyck C, Alessi C, et al. Clarifying confusion: the confusion assessment method. A new method for detection of delirium. Ann Intern Med 1990:113:941-8.

54 Anstey KJ, Cherbuin N, Herath PM, et al. A self-report risk index to predict occurrence of dementia in three independent cohorts of older adults: the ANU-ADRI. PLoS One 2014;9:e86141.

55 Burke WJ, Roccaforte WH, Wengel SP. The short form of the geriatric depression scale: a comparison with the 30-Item form. Topics in geriatrics 1991;4:173-8.

56 Ferraz MB, Quaresma MR, Aquino LR, et al. Reliability of pain scales in the assessment of literate and illiterate patients with rheumatoid arthritis. J Rheumatol 1990;17:1022-4.

57 Nicholls MER, Thomas NA, Loetscher T, et al. The Flinders handedness survey (Flanders): a brief measure of skilled hand preference. Cortex 2013;49:2914-26.

58 Üstün TB, Chatterji S, Kostanjsek N, et al. Developing the world Health organization disability assessment schedule 2.0. Bull World Health Organ 2010;88:815-23. 